\title{
Sprache aus Sicht des Ohrenzeugen oder citizen science
}

\section{Die Bandbreite des Laienwissens}

Zusammenfassung: Gerade wenn es um die Gewinnung und eine erste Bewertung von Forschungsdaten geht, ist derzeit oft vom Übergang zu citizen science die Rede. Nachdem dieses Konzept zunächst in den Lebenswissenschaften eine größere Rolle gespielt hat, findet es sich neuerdings auch in Teilen der Sprachwissenschaft. Viele einschlägige Initiativen schließen an die Tätigkeiten an, bei denen sich auch traditionell schon die professionalisierte Wissenschaft der Hilfe der ,Laien' bediente, sie können allerdings jetzt die in ungeahntem Ausmaß gewachsenen Möglichkeiten elektronischer Kommunikation und elektronischen Daten-Managements nutzen. Das digitale Interagieren erweitert die Möglichkeiten der als beteiligte „Laien“ gesehenen Personen aber doch so sehr, dass sich auch qualitativ ein neues Verhältnis zwischen den am Forschungsprozess Beteiligten entwickelt. In diesem Beitrag wird diskutiert, welche Folgen diese Veränderung für die wissenschaftliche Praxis, aber auch für das Verständnis des Konzepts „Wissenschaft“ hat.

Abstract: Especially when it comes to the collection and initial evaluation of research data, the transition to citizen science is often mentioned. After this concept played a major role in the life sciences, it is now also found in parts of linguistics. Many relevant initiatives follow on from the activities in which even professionalised science traditionally used the help of 'laymen', but they can now make use of the possibilities of electronic communication and electronic data management, which have grown to an undreamt-of extent. The digital interaction, however, expands the possibilities of the persons seen as participating "laymen" to such an extent that a new relationship between the participants in the research process develops qualitatively as well. This article discusses the consequences of this change for scientific practice, but also for the understanding of the concept of "science".

Eichinger, Ludwig M.: Direktor des Leibniz-Instituts für Deutsche Sprache (a. D.), Schopenhauerstraße 12, 68165 Mannheim, ludwig.eichinger@icloud.com 
Schlüsselwörter: Gesprochene Sprache, Erhebungsmethoden, Lexikographie, Dialektologie, Professionalisierung, citizen science light, citizen science proper, Spracheinstellungen, Online

\section{Materialität und die Intelligenz der Vielen}

\subsection{Die Ränder der Wissenschaft}

Bei Begriffen, die neu im Zentrum unserer Diskurswelt auftauchen, sollte man darauf sehen, welche Praxis damit aufgerufen wird (vgl. Eichinger 2016: IX-X) solch eine Überlegung und Behauptung passt, ohne den skeptischen Nebenton, $\mathrm{zu}$ der folgenden Bemerkung Ludwig Wittgensteins zum Funktionieren von Sprachspielen: „Begriffe können einen Unfug erleichtern oder erschweren; begünstigen oder hemmen“ (Wittgenstein 1977: 108).

Erkennbar nicht nur an der verdienstvollen Forschung, die in den letzten Jahren zur Wahrnehmungsdialektologie durchgeführt worden ist, sondern auch an der Zunahme von Methoden, die offenbar davon getragen sind und dazu dienen sollen, sich der ,Schwarmintelligenz' zu bedienen, hat die Beteiligung des „Laien“, des nicht professionell an einer Sache Interessierten am Forschungsprozess, unter dem Stichwort citizen science ein neues Gewicht erlangt. Bei aller Verschiedenheit der Dinge, um die es hier geht, haben sie doch alle damit zu tun, dass die veränderte Materialität der Kommunikation und die grundlegenden Verschiebungen in den Strukturen der Interaktion einerseits Möglichkeiten vernetzter Interaktion und der Vernetzung der Interaktion der Individuen geschaffen haben (vgl. Eichinger 2018: 70). Die Kommunikation in der digitalen Welt verändert die kommunikativen Möglichkeiten der Einzelnen, in einer Weise, die vielerlei scheinbar feststehende Grenzziehungen obsolet erscheinen lässt. Damit werden Eigenheiten, die vordigital als zentral galten, in ihrer Bedeutung reduziert. Das entscheidende Stichwort dabei könnte das der Vernetzung sein. Es trifft zu auf neue Mittel einer Art Gruppenkommunikation bzw. individueller Kommunikation mit nicht spezifischer Streuung der Information, es trifft auch zu auf die Vernetzung der Mittel, hinter der nicht nur die Unterscheidung von gesprochenen und geschriebenen Äußerungen zurücktritt, sondern bei der die Mehrmodalität eher zum Normalfall wird. Es trifft aber auch auf einer Art Metaebene zu, die technischen Möglichkeiten des Umgangs mit der Sprache und den damit verbundenen interaktionalen Praktiken erlauben nicht nur der professionellen Wissenschaft den Zugriff auf vorher nicht so festhaltbare oder analysierbare Ebenen und Phänomene der Interaktion, sondern 
geben auch dem interessierten Laien Einblicke und Beobachtungsmöglichkeiten, wie es sie so noch nie gab.

Andererseits und andersherum - von der vernetzten Welt aus gesehen wird auf diesem Weg der Zugriff auf eine Vielzahl von Daten ermöglicht, die in der elektronischen Welt zugänglich sind. Wenn solche Daten systematisch gesammelt und aufgearbeitet werden - ein anderes dieser catch words in modernen Narrativen ist Big Data -, sind Daten in einem Ausmaß und einer Differenzierung versammelt, dass sie neue Erkenntnis auf statistischer Basis und mit algorithmischen Prozeduren ermöglichen. Diese von mehrfachen Vernetzungen durchzogene Welt moderner Kommunikation lässt Wissen in ungeahnter Weise in erreichbare Nähe gerückt erscheinen, trägt damit zumindest potentiell und nach dem ersten Augenschein zu jener rationalen Entzauberung der Welt bei, auf deren virtuellen Charakter sich schon die in Max Webers Rede „Wissenschaft als Beruf“ angedeutete Potentialität beziehen lässt, dass die zunehmende Rationalisierung nicht „eine zunehmende allgemeine Kenntnis der Lebensbedingungen“ bedeute, sondern „das Wissen davon oder den Glauben daran, daß man, wenn man nur wollte, es jederzeit erfahren könnte“ (Weber 2002: 488). Die Voraussetzungen dafür zu schaffen, das ist seit ihrer Professionalisierung die Aufgabe der institutionalisierten Wissenschaft.

\subsection{Was der Bürger weiß - und wie man Nutzen daraus zieht}

Unter diesen Umständen der wissenschaftlichen Professionalisierung ist die Frage der zunehmenden Spezialisierung der fachlichen Praktiken und Diskurse auch eine Frage einer angemessenen Präsentation von Wissenschaft im gesellschaftlichen Raum. Die Anforderung, die Dinge jederzeit erfahren zu können, gewinnt zweifellos im Kontext der Individualisierung und unter den Bedingungen ansonsten immer ungehinderterer Interaktion an viel grundsätzlicherer Bedeutung. Der Zirkel des fachlichen Diskurses erscheint so als eine Instanz asymmetrischen Herrschaftswissens, dem gegenüber auch in Fragen der wissenschaftlichen Analyse so etwas wie ein herrschaftsfreier Diskurs eingefordert wird. Gerade die zunehmende Spezialisierung in der Wissenschaft stellt in dieser Hinsicht ein Problem dar. Daher war und bleibt dabei das Verhältnis von institutioneller Wissenschaft und der Beteiligung bzw. Interessen des ,normalen“ Bürgers mit seinen Erkenntnisinteressen unklar, galten sie doch eigentlich und im Kern als nicht wissenschaftlich. In der neuen digitalen Welt haben sich die Bedingungen der Erzeugung und Verbreitung von Wissen grundsätzlich verändert: Zugänglichkeit und Analysierbarkeit sind so viel weiter verbreitet, dass sich die Frage neu stellt, wie weit hier Erkenntnis aus der traditionell au- 
ßerwissenschaftlichen Welt in dieser als eine andere Art, aber als Wissenschaft verstanden werden könne. Die Frage dabei ist einerseits, wo und wie man wissenschaftliche von nicht-wissenschaftlichen Praktiken trennt, aber auch, ob es Fragestellungen und Bereiche gibt, wo sich die beiden Bereiche enger durchdringen, bzw. auch wo Dinge dem ,Laien“ und seiner Betrachtung und Betätigung nicht zugänglich sind (vgl. die - auffällig polemische - Diskussion in Finke 2016: 39-40).

Aufgrund der geschilderten gesellschaftlichen und kommunikativen Veränderungen ist es nicht überraschend, dass das Wort von der Bürgerwissenschaft, citizen science, einer ganz unterschiedlich ausgestalteten Beteiligung des Bürgers an der wissenschaftlichen Praxis, der nicht ein professioneller Wissenschaftler in diesem Gebiet ist, nicht nur in aller Munde ist, sondern nicht zuletzt auch seinen Platz im Förderungs-Leporello des Bundesministeriums für Bildung und Forschung (BMBF) gefunden hat. Auf den Seiten des BMBF ist im Kontext der entsprechenden Ausschreibung von 2016 eine Liste von den dreizehn geförderten Projekten aufgeführt, die aus über 300 Einreichungen ausgewählt wurden. Viele der Projekte sind lebenswissenschaftlich orientiert, ein Start-Projekt ist das der Kartographierung der Nachtigallen und ihrer Gesangtypen im Raum Berlin. ${ }^{1}$ Es ist in gewisser Weise auch ein prototypisches Projekt: Es betrifft ein Objekt, das sich traditionell oder auch aufgrund großräumigerer aktueller Diskurse eines breiten und auch emotionalen Interesses erfreut, es macht und verlangt keine größeren theoretischen Vorüberlegungen, und auch kein sehr komplexes Handlungs-Instrumentarium. Erkennbar ist auch, dass das Vorgehen im Rahmen eines Forschungskonzepts realisiert wird, dessen Rahmen von den Fragestellungen der institutionellen Wissenschaft gesetzt wird. So verweist auch das BMBF für seine Initiative erst einmal auf die Anerkennung und den verstärkten sowie modernisierten Einbezug der oftmals für den Erfolg unverzichtbaren unterstützenden Tätigkeit klassischer Wissenschaft, so heißt es in den Erläuterungen zu diesem Programm: „Abhängig von der genauen Fragestellung eines Forschungsprojektes können Bürgerinnen und Bürger mehr oder weniger leicht wichtige Daten zur Verfügung stellen.“2

Dass man hier unter den veränderten Bedingungen technischer Zugänglichkeit neue Möglichkeiten hat, ist gerade in nicht so technisierten geistes- und sozialwissenschaftlichen Kontexten offenkundig.

1 Vgl. die Darstellung des Projekts auf den BMBF-Seiten https://www.bmbf.de/de/dienachtigall-glaenzt-in-grammatik-8474.html (letzter Zugriff 01.06.2020).

2 https://www.bmbf.de/de/citizen-science-wissenschaft-erreicht-die-mitte-der-gesellschaft225.html (letzter Zugriff 18.03.2020). 
Es ist unter diesen Auspizien durchaus auffällig, dass unter den einigermaßen offiziell auffindbaren Projekten dieses Typs keines ist, das ein sprachwissenschaftliches Thema hätte. Warum ist das so, und woran könnte das liegen?

\section{Der Mund- und Ohrenzeuge, ein (verkappter) Bürgerwissenschaftler?}

\subsection{Vorbemerkung}

Man sollte denken, dass eigentlich die Voraussetzungen für bürgerwissenschaftliche Beteiligung an der Erforschung der sprachlichen Verhältnisse ganz gut wären: Es gibt ein vielfältig dokumentiertes Interesse einer allgemeinen Öffentlichkeit an sprachlichen Fragen, vor allem an Fragen der sprachlichen Korrektheit und Angemessenheit, von der Orthographie bis zur Frage der Fremdwörter, von Fragen der regionalen und staatsbezogenen Unterschiede bis zur Frage eines guten Standards. Zudem gibt es kaum eine andere Erscheinung des sozialen Lebens, die so sehr in die Domäne des Laien gehört: Er ist es, der spricht und schreibt.

Und auch die professionelle Sprachwissenschaft kommt ohne den, gerade auch ohne den linguistisch nicht verbildeten, Laien nicht aus. Besonders wenn man gesprochene Sprache untersuchen will, kommt man am Sprecher nicht vorbei. Es geht einem da allerdings so ähnlich wie dem an der Alltagsgeschichte interessierten Historiker, der es mit dem Zeitzeugen zu tun hat. Der Zeitzeuge ist zweifellos ein wertvolles Gut, er hat erlebt, was den Forscher interessiert, allerdings ist sein Interesse - zumeist - ein anderes als das des professionellen Historikers. Seine, des Zeitzeugen, Wahrheit ist für die Historie Material für eine im engeren Sinn wissenschaftliche Analyse. Trotzdem bleibt wahr, dass man an bestimmte Dinge nur herankommt, wenn man die Aussagen dessen, der dabei war, ernst nimmt.

\subsection{Der Sensor oder citizen science light}

Soweit gilt das auch für den Sprachwissenschaftler: Nicht nur wie der in den Wissenschaftsprozess einbezogene Partner empirischer Forscher, die Gewährsperson spricht, sondern auch, wie er sein Sprechen einschätzt und beschreibt, ist ein offenbar unverzichtbarer Beitrag zur Forschung. Wobei das nur zur Hälfte wahr ist: Eigentlich hätte man es lieber, wenn man ohne Mithilfe, ja ohne die 
Wahrnehmung des Sprechers sein Sprechen beobachten und aufzeichnen könnte. Das ist zweifellos schon grundsätzlich schwierig, noch schwieriger geworden unter der Bedingung, dass man von vorneherein das Einverständnis zur Beobachtung und Aufnahme sprachlicher Daten einholen muss. In gewisser Weise haben diese Regeln zum Daten- und Personenschutz die Personen, die wir davor im Prinzip als Quellen objektsprachlicher Äußerungen betrachtet haben, damit ohnehin schon zu aktiv Mitwirkenden im wissenschaftlichen Prozess gemacht.

Es gibt daneben schon länger Versuche, durch eine stärkere Aktivierung der Beteiligten (der ,Gewährspersonen') in der Erhebung der Daten den störenden Faktor des professionellen Wissenschaftlers auszuschalten. Ein frühes sprachwissenschaftliches Experiment in diesem Sinne war, dass im sogenannten ErpProjekt von Werner Besch und Klaus Mattheier (vgl. z. B. Besch 1997) die Aufgenommenen gleichzeitig zu Aufnehmenden eines von den Forschern lediglich initiierten Gesprächs zwischen den Gewährspersonen wurden. Das ist eine erste Stufe auf dem Weg des Mund- und Ohrenzeugen, also von dem, der selbst die gewünschten Formen, Äußerungen und sprachlichen Handlungen produzieren und aufgrund seiner eigenen Wahrnehmung dazu beurteilende Äußerungen abgeben kann, hin zu einer professionalisierten Rolle. Was das mit der Interaktion der Beteiligten macht, sei hier beiseitegelassen, die Alternative einer weithin als ,teilnehmende Beobachtung benannten Methodik versucht das Problem von der anderen Seite, dem Beobachtenden her anzugehen und dessen Sichtbarkeit zu reduzieren, was für den vorliegenden Beitrag vom Thema abführt. Das hier vorläufig angedeutete methodische Dilemma hat in unterschiedlichen Forschungssettings unterschiedliche Konsequenzen. Hier sei nur auf eine Folge hingewiesen, die in der Fortsetzung des Weges auf eine zunehmende Professionalisierung des beteiligten ,Laien“ liegt. Sie betrifft insbesondere Aspekte einer Gesprächsforschung, die zu einer möglichst diversifizierten Sicht ihres Objektbereichs kommen möchte. Dabei stellt sich zum einen die gerade schon angesprochene Frage, wie man hier zu quasi-natürlicher Objektsprache kommt, und das einmal unter diesen generellen Bedingungen, zum anderen spezifischer, wenn es in einer Typologie möglicher Gesprächskonstellationen um Situationen geht und man zu Konstellationen kommt, die externer Beobachtung nicht zugänglich sind. Als ein Beispiel dafür, wie man dieses Problem mit Aktivierung von Laienforschung lösen möchte, kann man die 2019 gelaufene Akquisitionsphase für die Datenbank FOLK (Forschungs- und Lehrkorpus) im Rahmen des Projekts Gesprochene Sprache am Leibniz-Institut für Deutsche Sprache (IDS) 
betrachten. Im Sinne einer möglichst breiten Streuung der Gesprächstypen werden in der Ausschreibung „Quasselstrippen und Hobbyfilmer“33 gesucht. Um die Hürden der Zusage möglichst gering zu halten, wird in einem beiläufig alltäglichen Ton an ein vorwissenschaftliches Interesse am Sprechen und an der technischen Aufnahme von Bild und Ton appelliert. „Du bist kommunikativ und sprachinteressiert?“ heißt es da unter anderem. Die sprachlichen ,Zeitzeugen“ mit ihrer Vernetzung, ihrer spezifischen lebensweltlichen Kompetenz und ihren modernen technischen Optionen lassen in diesem Text ein Bild der Bürgerbeteiligung an einem sie selbst auch interessierenden Objekt aufscheinen.

Eigentlich werden mit der Aufforderung in diesem Text zwei Aspekte gleichzeitig angesprochen. Zum einen geht es um die Frage, wie man zu einer möglichst hohen Streuung und zudem zu einer balancierten Verteilung und guten Subdifferenzierung der Interaktionsdomänen kommt. Die technischen Möglichkeiten erlauben es, diese Erhebungen über Crowdsourcing zumindest anzureichern, was mengenmäßig effektiver und prinzipiell vielfältiger ist, als das über traditionellere Erhebungsverfahren in absehbarer Zeit der Fall wäre. Das ist für die Korpuserstellung zweifellos ein vernünftiges Ziel, das das Korpus größer und im Hinblick auf die erfassten Lebensbereiche differenzierter erscheinen ließe. Wenn man eine neue Beschreibung zum Bestand des FOLKKorpus ansieht (vgl. Kaiser 2018), überrascht es nicht, dass in der Ausschreibung zum Beispiel auf „Bedarf“ für den Bereich Dienstleistungen hingewiesen wird. Auf der anderen Seite kann man auch vermuten, dass manche größeren Lebensbereiche mangels hinreichender Differenzierungsangebote so relativ ungegliedert erscheinen.

3 http://agd.ids-mannheim.de/bilder/Aufruf_FOLK.pdf (letzter Zugriff 18.03.2020). 


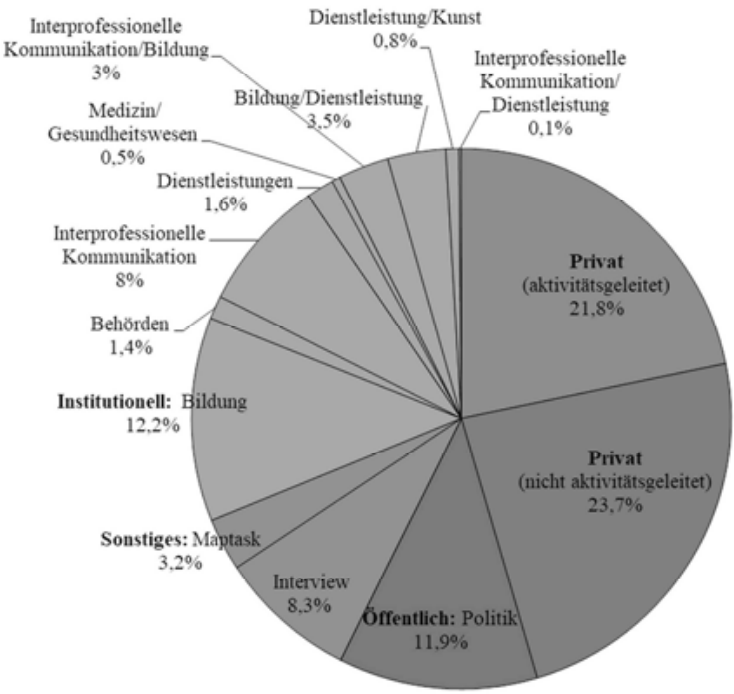

Abb. 1: Lebensbereiche (Kaiser 2018: 531).

Neben dieses ökonomische Argument tritt ein zweites, das eine auf umfassende Abdeckung von Lebensbereichen zielende Sammlung zumindest von bestimmten forschungsethischen Problemen befreit. Nicht umsonst heißt es so nebenher: „und ,nie genug' bekommen wir von Tischgesprächen in der Familie und Telefongesprächen“4. Tatsächlich bietet die Möglichkeit, die Erstellung professionell brauchbarer Erhebungen an eine beteiligte Person, oder zumindest einen vertrauten Interaktionszeugen, zu übergeben, die Chance, Lebensbereiche dokumentiert zu bekommen, die aus forschungspraktischen oder forschungsethischen Erwägungen einer externen Exploration nicht oder kaum zugänglich sind. Das stellt durchaus einen bemerkenswerten Schritt im Hinblick auf die Stratifikation eines solchen Korpus dar, die Ergebnisse dieses Tuns stehen auch allen zur Verfügung, die in diesem Bereich forschen wollen, aber alles findet doch unter der Ägide professioneller Wissenschaftsvorstellung statt.

Wenn es konkret wird, wird das auch im Text schon klar, wenn auch in ironischem Ton gehalten (,Du hättest Spaß daran, einmal Aufnahmeleiter zu sein?“), dass die Fähigkeiten eines „Aufnahmeleiters“ gesucht werden, der mit

4 http://agd.ids-mannheim.de/folk.shtml (letzter Zugriff 18.03.2020). 
gewisser technischer Unterstützung bei bestimmten Gesprächskonstellationen eine relativ eigenständige Funktion im Forschungsprozess einnehmen soll. Das könnte man vielleicht schon als eine gehobene Stufe von citizen science betrachten. Wobei noch interessant wäre, zu erfahren, welche fachliche Vorbildung Personen tatsächlich haben, die sich auf diese Ausschreibung melden. Wichtiger ist aber vielleicht ein anderer Punkt: Im Hintergrund steht hier etwas, was am Anfang so beiläufig mit dem Stichwort von der Materialität der Kommunikation angesprochen wurde. Gerade an dieser Stelle haben sich die Grenzen der Möglichkeiten zwischen Laien und professionell Daten Erhebenden deutlich geändert. Was verändert sich am Status der mitarbeitenden ,Laien‘, wenn durch den Fortschritt der Technik beim Crowdsourcing ein medial kompetenter Laie Aufnahmen von professioneller Qualität selbst erzeugen kann? Und nicht nur das, verschiedene offenzugängliche Programme und Apps erlauben eine automatische Voranalyse, die weit in das eingreift, was man immer schon als einen wichtigen Punkt wissenschaftlicher Arbeit betrachtet hätte.

Ein Beispiel dafür sind Kartographierungsprojekte, wo durch die Kombination der vorsortierten Informationen sozusagen automatisch eine höhere Genauigkeit erreicht wird. In diese Richtung gehen etwa die dezidiert als citizen science-Unternehmungen ausgeflaggten Erhebungen zu mehrsprachigem Linguistic Landscaping wie das Luxemburger Lingscape-Vorhaben (vgl. z. B. Purschke 2018). Es nutzt den Charme der Nutzung einer App und der immer besser gewordenen Foto-Funktion auf den Smartphones, wodurch ein einfacher Zugang mit einer geringen Hürde auch zu sonst schwer erreichbaren ,Exploranden“Gruppen erreicht wird. Allerdings ist damit auch eine gewisse Unsicherheit in Bezug auf die Zusammensetzung der Population verbunden, auf die sich die erhobenen Daten stützen. Das betrifft vermutlich weniger einen gewissen Alters-Bias, als vielmehr auch die Tendenz zu interessegeleiteter Datenlieferung in solchen Interaktionen. Man kann allerdings, wie Christoph Purschke (2018: 70) anmerkt, darauf hoffen, dass diese potentiellen Mängel „in der Regel durch die schiere Menge des Materials“ aufgewogen würden. Dennoch stellt sich auch hier die Frage, wo die Grenze zwischen einer technisch avancierten Materialerhebung und -auswertung, auch der Bewertung aus Sicht des involvierten Augen- und Ohrenzeugen, in gewisser Weise wissenschaftlichen Unterstützungstätigkeiten, bei von der professionellen Wissenschaft geprägten Fragestellungen und eigenständiger Bürgerforschung verläuft. Auf der Internet-Präsentation des Projekts sind vier Stufen der Teilnahme bzw. Aktivitäten angegeben: signs (fotografisches Erfassen und Hochladen), map (sich informieren auf den entstehenden Karten), projects (Nutzung des Werkzeugs/der Daten für eigene Vorhaben) und administration (Nutzung des Werkzeugs zur Datenordnung). Von 
ihnen sprechen die letzten beiden Optionen projects und administration eine Zielgruppe bzw. einen Tätigkeitsbereich an, die in der Schnittmenge zwischen der klassischen Wissenschaft und neuen Beteiligungsformen verläuft. Wobei sich offenbar die Frage stellt, wie weit die neue Technik einen qualitativen Schritt in der Beteiligung darstellt oder eine Erweiterung der ,Sensor'-Funktion. Klar ist schon, dass hier durch die Menge und Distribution der Daten ein vollständigeres Bild entstehen kann. Es ist zweifellos nur ein zeitgebundenes Bild in der doch noch frühen Phase solcher Projekte, wenn man feststellt, dass die genannten Anschlussprojekte ${ }^{5}$ derzeit im Wesentlichen ,(Nachwuchs)Wissenschaft proper' bzw. anwendungsorientierte Nutzungen (z. B. didaktischer Art; vgl. auch Purschke 2018: 72-74) darstellen.

\subsection{Der Experte}

Es gibt allerdings traditionsgemäß, zum Beispiel bei den Historikern, den engagierten Laien-Historiker, der häufig zu einem ihn interessierenden Bereich eine außerordentlich spezifische Kenntnis besitzt, und sich dort mit hohem Engagement einsetzt - verständlicher- und wohl auch vernünftigerweise, ohne sich allzu sehr mit den methodischen und theoretischen Frontlinien der professionellen Wissenschaft zu beschäftigen.

Auch die Sprachwissenschaft, in Sonderheit die Dialektologie, wenn man so will die Mutter der Beschäftigung mit der gesprochenen Alltagssprache, kennt traditionell ein starkes Laien-Interesse, das sich zum Beispiel an der Beschäftigung mit dem Wortschatz zeigt. Das macht sich die Dialektlexikographie (wie die Lexikographie insgesamt) auch zu Nutze. Und so findet man denn zum Beispiel in den Listen der Gewährspersonen für das Wörterbuch der Bayerischen Akademie Personen mit den verschiedensten Berufen, so zum Beispiel im Geburtsort des Verfassers dieses Beitrags für die letzten Erhebungsrunden (= II und III) die folgenden Personen, die bei den Umfragen des Wörterbuchs Material liefern:

Gerstl, Helga, Gerichtsvollzieherin III; Grätzer, Hans, Oberlehrer II; Hummel, Franz, Dr., Arzt III; Kitzinger, Ludwig, Rektor III; Marterer, Gg. II; Schachtner, Franz Xaver, Landwirt III.

(https://bwb.badw.de/materialsammlung/sam mlerverzeichnis.html; letzter Zugriff 18.03.2020).

5 https://lingscape.uni.lu/\#projects (letzter Zugriff 01.06.2021). 
Offenkundig sind hier die Augen- und Ohrenzeugen sprachinteressierte Zeitzeugen und gleichzeitig Experten für verschiedene Aspekte des wissenschaftlichen Feldes. Das geht zum Teil den Zugang zu verschiedensten Bereichen des sprachlichen Umfelds an, etwa bei Gerichtsvollzieher und Arzt, teils repräsentieren sie in ihrem sprachlichen Umfeld den Kernbereich der Untersuchung, teils haben sie beruflich mit dem sprachlichen Umfeld zu tun (Rektor, Oberlehrer). Was das Ziel der Befragung, den Wortschatz angeht, sind sie über ihre alltagssprachliche Kompetenz hinaus durchaus Experten in ihren professionellen Handlungsbereichen; zum Teil mit einer Spezifik der Kenntnis vor allem auf der Ebene der Wörter und Sachen, wie sie kaum einem der hauptberuflichen Lexikographen zu eigen sein dürfte. Das Bayerische Wörterbuch der Bayerischen Akademie der Wissenschaften zeigt auf seinen Online-Seiten etliche Beispiele, die das eindrücklich bestätigen. ${ }^{6}$

Natürlich ist da ein Interesse an der Sprache und ihrer Rolle für die Lebenswelt in den eigenen Räumen, aber es ist wohl immer noch das, was die citizen-science-Gemeinde mit dem Stichwort des Bürgers als Sensor benennen würde - was zweifellos einen wichtigen Schritt beim wissenschaftlichen Erkenntnisprozess in diesem Bereich wissenschaftlicher Tätigkeit darstellt. Es ist das andererseits eine jener Stellen, an denen der Informant nicht nur sprechend belegt, wie er spricht, sondern was er über sein Sprechen und über die besprochenen Sachverhalte und Praktiken weiß. Er ist daher auch in diesem Kontext Experte für bestimmte Aspekte der wissenschaftlichen Tätigkeit, womöglich auch als in einem anderen Fach wissenschaftlich tätige Person. Auf die damit berührten Fragen von Interdisziplinarität und gegenseitiger fachlicher Unterstützung soll hier nicht weiter eingegangen werden. Aber auch so wird es an dieser Stelle ohnehin schon komplex. Und zwar ganz grundlegend, scheint es doch so, dass nicht nur die kategoriale Abgrenzung von wissenschaftlichem und ,laienhaftem' Vorgehen nicht so recht zu ziehen ist, und dass sie zudem überlagert wird von dem funktionalen Begriff des Experten, der Expertise. Man hat mit den verschiedensten Überschneidungen zwischen diesen Zuordnungen zu rechnen. Manches hängt schon an der wissenschaftssystematischen Bestimmung von Wissenschaft als Beruf, mit einem einforderbaren Bezug auf die jeweilige Wissenschaft mit ihrem professionellen Hintergrund, und mit dem, was man mit Wendungen wie der von so etwas wie situationsentbundenem Interesse $\mathrm{zu}$ fassen versucht. Gerade bei Sprache gibt es einen merklichen Unterschied zwischen dem Wissen des Beteiligten über die Verhältnisse der eigenen Sprachwelt, und seiner unzweifelhaft als wissenschaftlich betrachteten Analy-

6 https://bwb.badw.de/materialsammlung/beispiele.html (letzter Zugriff 18.03.2020). 
se. Natürlich ist das nicht nur ein Problem des sogenannten Laien, auch die Beschäftigung des professionellen Sprachwissenschaftlers mit der eigenen Sprachwelt hat mit diesem doppelten Blickwinkel $\mathrm{zu}$ tun. Tatsächlich ist das Wissen über die eigene Sprache eine Sache eigener Art, man befindet sich in komplexen objekts- und mehrfach gestuften metapragmatischen Verhältnissen, und die sprachlichen Verhältnisse sind nicht so leicht von den eigenen Handlungen ablösbar. Das kommt in Roman Jakobsons berühmten Formulierungen zu diesem Punkt nur ansatzweise zum Ausdruck, es ist nicht zweimal dieselbe metasprachliche Funktion, die hier benannt wird:

Doch die Metasprache ist nicht nur ein unabdingbares wissenschaftliches Werkzeug für Logiker und Linguisten; sie spielt auch eine wichtige Rolle in unserer Alltagssprache. [...] Wenn der Sender und/oder der Empfänger kontrollieren wollen, ob beide denselben Kode gebrauchen, orientiert sich die Rede am KODE: sie vollzieht eine metasprachliche (d.h. erläuternde) Funktion.

(Jakobson 2019: 177)

Dennoch gilt natürlich, dass die Differenz zwischen den Aussageweisen lediglich die Interpretierbarkeit von Meta-Äußerungen erschwert, da sie in den jeweilig gehörigen Rahmen gestellt werden müssen, dass das aber nicht das Prinzip erschüttert, Äußerungen von Sprechern ernst zu nehmen. Die Selbsteinschätzung von Sprechern ist ein Faktum, dem man an seinem Ort trauen sollte:

Eigentlich ist das gar keine Frage. Da nicht alle Sprecher Lügner sind, wäre es sogar unklug, den Sprechern nicht zu trauen. Allerdings variieren die Urteile über die gleichen sprachlichen Phänomene in überraschender und daher manchmal auch amüsanter Weise.

(Eichinger 2010: 433)

Natürlich bedarf es einer spezifischen Vorsicht, in Eichinger (2010) werden dazu drei Punkte zum Charakter unoffensiver Kommunikation zwischen Linguisten und ihren in dieser Hinsicht nicht-professionellen Gesprächspartnern aufgeführt, von denen an dieser Stelle die zweite die wichtigste ist:

Zum ersten: Wenn man die Menschen nach ihrer Sprache und ihrem Sprachgebrauch fragt, bekommt man Meinungen zur Antwort.

Zum zweiten: Die metasprachlichen Äußerungen von Sprechern, die keine professionellen Sprachwissenschaftler sind, sind oft nicht leicht analytisch einzuordnen.

Zum dritten: Normale Sprecher sind keine Lügner, aber sie versuchen, zu ihrem Gesprächspartner nett zu sein.

(Eichinger 2010: 433)

Es kommt dann darauf an, was man daraus macht. Und das nicht einmal so sehr in jenen Fällen, wo wir es mit relevanzbedingten Fehleinschätzungen und 
entsprechenden Fossilisierungen im kommunikativen Gedächtnis zu tun haben, wie bei den Narrativen über den Zusammenhang der deutschen Dialektlandschaft, der unter anderem die wahrnehmungsdialektologischen Forschungen an der Universität Kiel nachgegangen sind, und wo sich unter anderem zeigt, dass es nicht unerheblich ist, aus welchem sprachlichen Areal man selber kommt, wenn man den gesamten Raum des Deutschen in der Bundesrepublik Deutschland untergliedern soll (diese und andere Fragen werden ausführlich in Hundt, Palliwoda \& Schröder 2017 dokumentiert). Auch in entsprechenden Studien im Rahmen eines größeren Spracheinstellungsprojekts am IDS ergaben sich ganz ähnliche Befunde, die sich auch im Hinblick auf den Übergangsbereich vom Laien- über ein spezifisches Experten- hin zu einem fachlich gestützten Wissen interpretieren lassen.

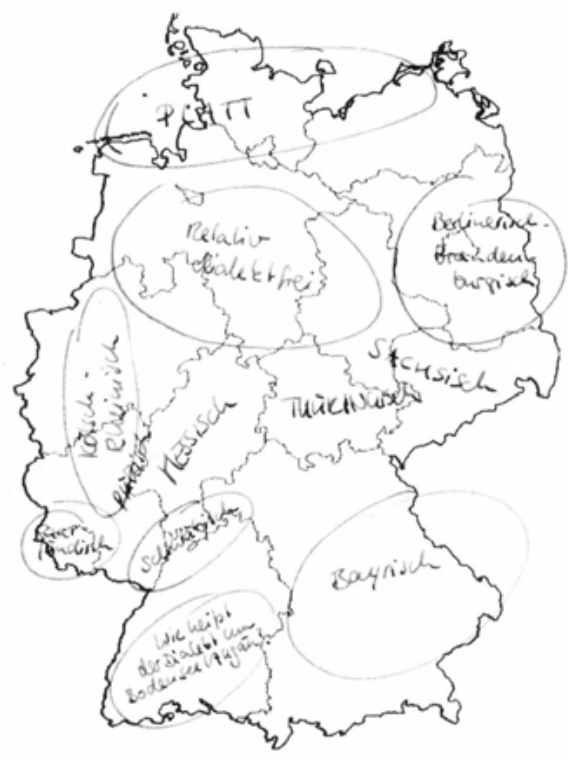

Abb. 2: Kölner Gewährsperson (vgl. Plewnia \& Rothe 2012: 73). 


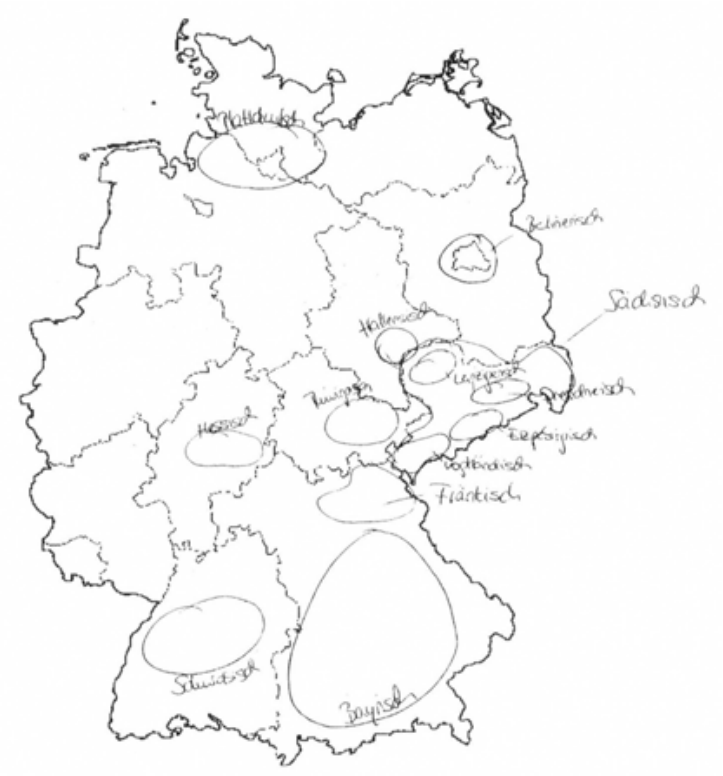

Abb. 3: Leipziger Gewährperson (vgl. Plewni \& Rothe 2012: 93).

Die Verzerrung ist augenfällig, aber anamorphotische Karten sind ja auch in anderen Zusammenhängen erhellend; besonders und allerdings, wenn sie auf großen Datenmengen und der unterschiedlichen Primärsetzung von bestimmten Metadaten beruhen. ${ }^{7}$ Und auch wenn wir die Aussagen der die Daten liefernden Laien ernstnehmen, werden sie zu wissenschaftlichen Daten doch erst, wenn sie Gründen genügen, die als Elemente eines fachlichen WissensKonzeptes angenommen werden können. So würden vermutlich viele auf solche Weise befragte Personen eher sagen, sie glaubten etwas zu wissen als sie wüssten es. Hier sind sie eher Experten ihrer selbst, so dass sie einerseits als Zeugen ihrer sprachlichen Weltsicht betrachtet, andererseits im Vergleich zu der wissenschaftlichen Standardcharakterisierung regiolektaler Verteilung im Deutschen beurteilt werden. Man wird aus den verschiedenen andersartigen Strukturierungsvorschlägen keine Herangehensweise herauslesen wollen, die man als andersartiges Konzept von wissenschaftlicher Betrachtung der Sprachräume bezeichnen würde. Wiewohl das ein - externer - wissenschaftlicher Blick

$7 \mathrm{Zu}$ einer entsprechenden Raumbeobachtung - raum- vs. bevölkerungsbezogen - s. etwa https://bbsr-geodienste.de/raumbeobachtung/ (letzter Zugriff 18.03.2020). 
durchaus versuchen könnte. Was die beiden oben abgebildeten Karten (vgl. Abb. 2 und 3) gemeinsam haben, ist ja, dass sie einerseits durchaus einen Blick für die Gesamtstruktur haben. Es gibt in beiden Karten offenbar eine eher sozialpsychologisch fundierte Grundidee von einem Süden, der irgendwie von der Vorstellung von zwei großen Dialekträumen geprägt ist, einem den äußersten Norden kennzeichnenden Plattdeutsch, dem Berlinischen als typischer Stadtsprache und einem recht großen ,aregionalen' Raum in der nördlichen Mitte, und einen Streifen ungewisser, aber merklicher regionaler Differenzierung in der Mitte, an der jeweiligen politischen Gliederung orientiert. In beiden Fällen zeigt sich eine spezifischere Art der Kategorisierung im jeweiligen Nahbereich, das Kölnisch-Rheinländische in dem einen Fall als eine Art Gegenpol zum Berlinisch-Brandenburgischen, die ganz kleinteilige Untergliederung des Sächsischen im anderen Fall, mit dem Dresdnerischen, dem Hallensischen und dem Leipzigischen als prägenden Stadtsprachen. Es sind zudem die Karten von Menschen, die aus Räumen stammen, in denen sprachliche Regionalität jedenfalls eine gewisse Rolle spielt, in anderen Fällen - Bielefeld - sehen Abdeckung und räumliche Zuordnung deutlich schwächer aus. Man sieht hier, dass man dem sprachlichen Zeitzeugen hier symptomatische Kenntnisse zubilligt, die einer gewissen Reflexion auf Fachliches bedürfen, aber eigentlich nur über professionelle Fachlichkeit analysiert werden kann, wobei man vermuten muss, dass die sprachliche Zeitzeugenkompetenz eher relativiert wird, wenn sie von eigener Wissenschaftlichkeit überlagert wird.

\section{3 citizen science proper?}

\subsection{Was der Bürger weiß}

Es muss einem klar sein, dass diese Bereitschaft zur Unterstützung des wissenschaftlichen Prozesses häufig eher auf allgemeiner Freundlichkeit basiert, der Bereitschaft, jemandem zu helfen, oder auch dem Gefühl, irgendwie funktional dazu verpflichtet zu sein, über das eigene Sprechen Auskunft zu geben, ohne allzu viel mit den wissenschaftlichen Zielen des Linguisten gemein $\mathrm{zu}$ haben, dessen prinzipiell doch sehr grundlagenorientierte Sichtweise wenig mit Praktiken und Erfahrungen mit Sprachspielen bei den sogenannten Laien zu tun hat. Dass das zu metapragmatischen wie praktischen Wirrnissen führen kann, sei anekdotisch anhand eines zum literarischen Autor gewordenen Linguisten und dessen Schilderung seines wissenschaftlichen coming of age angedeutet. Er beschreibt, wie er versucht, seinen Onkel im Sinne der dialektologischen Wen- 
ker-Sätze zu interviewen, deren erster in der im süddeutschen Raum verwendeten Fassung lautet: Im Winter fliegen die trocknen Blätter durch die Luft herum.

Wenninger streikte aber gleich beim ersten Satz: Im Winter fliegen die trockenen Blätter in der Luft herum. So einen Unfug könne er nicht nachsprechen, noch dazu, wo ich alles auf Tonband aufnähme, alles sträube sich in ihm dagegen. [...] im Innviertel liegt im Winter der Schnee, und die Blätter fliegen hierzulande im Herbst in der Luft herum.

(Brandstetter 1982: 108-109)

Nicht immer erscheint das Ansinnen der Wissenschaft so fremd von den eigenen Vorstellungen vom Nutzen einer bestimmten (sprachlichen) Praxis. Es gibt Abstufungen. Die Idee der Wenker-Sätze war ja im Kern auf die lautlichen Verhältnisse bezogen. Gerade aber Fragen des Wortschatzes, auch der festen Wendungen, scheinen dem gegenüber vergleichsweise unproblematisch zu sein, so gibt es denn auch durchaus Wörterbücher von Laien, die aber eigentlich nicht auf dem Markt der Wissenschaft konkurrieren wollen, sondern in gewisser Weise eine - häufig identitätsbezogene und dann eben nicht so situationsentbundene - Anwendungsperspektive realisieren.

Es ist nicht untypisch, dass solche Initiativen zum Beispiel an eher volkskundliche Institutionen angeschlossen sind; nicht umsonst sind klassische Dialektatlanten ein analoges Paradebeispiel für die Überschneidung der fachlichen Interessen am analogen Objekt. Es seien nur zwei Beispiele aus dem süddeutschen Raum kurz erwähnt, die für dieses Phänomen stehen. Das Bezirksmuseum von Buchen im Odenwald entwickelt seit einigen Jahren unter dem Titel ,SprachRaum. Das Sprachmuseum im Odenwald“ eine analoge, im Museum vorfindliche, aber auch mehr und mehr digitale Version einer Dokumentation der sprachkulturellen Verhältnisse. „Das Projekt“ so heißt es dort, „ist

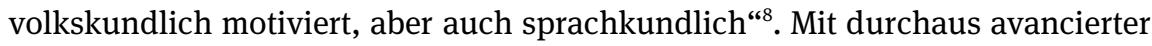
Technik wird eine ,sprechende“ Kartendarstellung des einschlägigen Raums erstellt, in die neuerdings auch über eine App per Smartphone Beispiele eingegeben werden können, bei denen auch über Transkriptionen Hilfe bei der Analyse und Verständlichkeit geleistet und über Quiz-Formen ${ }^{9}$ Verbesserungen der Zuordnung geleistet werden können. Hier wird von interessierten Laien eine ganze Reihe der Tätigkeiten im Rahmen eines von den Wissenschaftlern konzipierten Projektziels übernommen. Sie sind von einer Art, wie sie in gängigen Überlegungen zum Konzept citizen science auch auftauchen, und in den obigen Beispielen zum linguistic landscaping schon als Bestandteile zu erwähnen wa-

8 https://www.sprachraum.de/das-projekt/ (letzter Zugriff 18.03.2020).

9 https://www.sprachraum.de/sprachraum/quiz/ (letzter Zugriff 18.03.2020). 
ren. Die linguistische, in diesem Fall weithin dialektologische, Basis wird aus den gängigen sprachwissenschaftlichen Analysen genommen, man befindet sich im Kontakt mit einschlägigen universitären Instituten. Dieses in sich ganz eindrucksvolle gelungene Beispiel mag vielleicht als typisch für eine anwendungsbezogene Laien-Beschäftigung mit fachlich ordentlichen Mitteln aus verschiedenen Bereichen gelten, die moderne Möglichkeiten zur Vermittlung in einem konkreten Rahmen nutzt - hier das Dokumentationsinteresse der regionalen Sprachlandschaft für den an der lokalen Geschichte und Identität Interessierten. Wie weit das Instrumentarium der institutionellen Wissenschaft in solchen Projekten genutzt wird, mag variieren, die damit verbundenen Intentionen werden von Seiten der Wissenschaft eigentlich nicht als zusätzliche wissenschaftliche Fragestellungen oder Herangehensweisen angesehen. In unseren Fall zeigt der Vergleich mit anderen sprechenden Sprachatlanten, etwa den Sprachatlas Baden-Württemberg ${ }^{10}$ mit seinen Vorbildern, der ,Sprechende Sprachatlas von Bayern und der Sprechende Sprachatlas von BayerischSchwaben'11, um nur einige zu nennen, dass bei ihnen, obwohl sie ihrerseits schon als (sprachwissenschaftliche) Popularisierungen der Ergebnisse dialektologischer Forschung zu gelten haben, professionelle Fachlichkeit eine weitaus prägendere und in der Präsentation unmittelbar sichtbare Rolle spielt.

Dem Selbst- wie Fremdbild eines intensiveren Laien-Interesses zwischen ethnologischen und linguistischen Wahrnehmungen entspricht etwa ganz explizit ein im Internet zugängliches Bairisches Wörterbuch ${ }^{12}$, das von einem ebenfalls volkskundlich bewanderten Autor verfasst wurde.

abgebn obgebn

Aussprache: [å:gem / åbgem]

abgebn - obgebn - obgebm - obgeem - abgeem - abgebm - abgeben

abgeben, übergeben, aushändigen (...i muaß mei Krankmeldung obgebn!)

sich beschäftigen mit etwas, sich befassen mit etwas/jdm. (...mit so am Gschwerl mog i mi ned abgebn!)

Das Partizip Perfekt von abgebn/obgebn lautet abgebn/obgebn [å:gem / åbgem] = abgegeben (...d' Heigl Resi hod anLeffe abgebn!)

$10 \mathrm{https}$ //escience-center.uni-tuebingen.de/escience/sprachatlas/index.html\#8/48.674/ 8.989; (letzter Zugriff 18.03.2020).

$11 \mathrm{https} / /$ www.bayerische-landesbibliothek-online.de/sprachatlas (letzter Zugriff 18.03.2020).

12 https://www.bayrisches-woerterbuch.de/ (letzter Zugriff 18.03.2020); Schreibungen so im Netz. 
Wenn man beliebige Einträge wie den für abgeben ansieht, bemerkt man, dass der auffällige Wortschatz, das als typisch Angesehene im Mittelpunkt steht, dass die Erläuterungen allerdings gewisse Grundinformationen auch sprachwissenschaftlicher Art liefern, die vom Status eines (sich selbst nicht ganz ernstnehmenden) Laien-Experten sprechen. Der auffällige Wortschatz, die Aussprachevariation, eigentlich nebenher das Redensartliche ergeben ebenfalls ein Bild eines populären Laien-Experten. Natürlich würde man bei einer solchen, von spezifischen Popularisierungsinteressen gesteuerten Aktivität und ihren Ergebnissen nicht von Bürgerwissenschaft reden.

Allerdings ist es sicherlich kein Zufall, dass auch Projekte, die sich bemühen, die Expertise der nicht in der professionellen Wissenschaft tätigen Personen, die an der Sprachwissenschaft interessiert sind, in eine Art bürgerwissenschaftlichen Engagements hereinzuholen, zumeist ebenfalls eher an den Wörtern, den Redewendungen, auch noch an gewissen grammatischen Auffälligkeiten ansetzen. Um an dieser Stelle nicht immer den Atlas der deutschen Alltagssprache zu bemühen, seien hier einmal die auch in diesen Forschungszusammenhang gehörende schweizerische Dialekt- $\mathrm{pp}^{13}$ und ihr größeres Nachfolgeprojekt (vgl. Leemann, Derungs \& Elspaß 2019) als Beispiel herangezogen, die mit einer spielerischen automatischen Einordnung anhand von lexikalischen, aber auch ,aussprachemäßigen' Varianten beginnt, und eigene Korrekturen zulässt (vgl. Brinckmann et al. 2008: 3189; Kleiner 2015: 497). Infolge der CrowdSourcing-Technik ergeben sich hier dramatische Datenmengen, die für bestimmte Fragestellungen eine Dichte der Dokumentation erzeugen, wie sie mit traditionelleren Methoden nicht denkbar ist. In Leemann, Derungs \& Elspaß (2019: 3) wird ausgeführt:

[...] Leemann. et al. began developing mobile and web-apps which enable crowdsourcing of regional language data from speakers through a quiz [...]. The users go through this quiz on regional language use and, at the end of the quiz, the app tells them where they are from, based on their linguistic features. [...] The user can then evaluate the quiz result, provide metadata on age and gender, and submit this data.

Dieses Befragungsinstrument, das über Spiegel Online und den Tagesanzeiger online präsentiert wurde, wurde von 2015 bis 2019 von 1,9 Millionen Menschen genutzt, zudem mit einer Verteilung nach verschiedenen Metadaten (Alter usw.), die nicht dramatisch einseitig erscheint. Wie auch die erzielten Ergebnisse, die in Leemann, Derungs \& Elspaß (2019) präsentiert werden - Veränderun-

$13 \mathrm{https} / /$ www.schweizforscht.ch/projekte/projektarchiv/dialaekt-aepps (letzter Zugriff 01.06.2021). 
gen im Vergleich zu und seit Jürgen Eichhoffs Wortatlas der deutschen Umgangssprachen (1977-2000) - zeigen, sind die Ergebnisse solcher neuen webbasierten und Apps nutzender Studien bemerkenswert. Hier kommt es allerdings mehr darauf an, dass die Nutzer dieser Apps auf diesem Weg über den Status ,reiner' Ohrenzeugen und Experten ihrer selbst hinaus aktiviert werden, so dass hier möglicherweise über die neue Medialität ein qualitativer Sprung gemacht wird, der den Nutzer in gewissem Umfang zum Bürgerwissenschaftler macht.

\subsection{Was der Bürger wissen will}

Auffällig dabei ist, dass diese Aktivierung - vielleicht auch den medialen Gewohnheiten entsprechend - über den Reiz eines Spiels getriggert wird. Das ist ganz offenkundig eine in diesem Kontext gängige Herangehensweise. So wurde zum Beispiel am IDS in eher vermittelnder Funktion für das Forschungsschiff im Jahr der Geisteswissenschaften (2007) vom Projekt Deutsch das Spiel Hör mal, wo der spricht, entwickelt, bei dem auch schon ein gewisses Interesse an der eigenen sprachlichen Einordnung als Heranführung an sprachwissenschaftliche Interessen verstanden wird (vgl. Brinckmann et al. 2008: 3189; Kleiner 2015: 497). In Leemann, Derungs \& Elspaß (2019: 23) werden weitere Beispiele genannt.

Offenkundig handelt es sich bei dieser Art von Vorgehen um eine grundlegende methodologische Erweiterung bei Vorhaben, die auf flächendeckende Erhebung von Daten dieses Typs zielen. Sie leben außer von den technischen Möglichkeiten ganz grundsätzlich davon, dass sie die Interaktion mit den Laien einbauen. Nicht zuletzt aufgrund der kompetenten Handhabung des technischen ,Handwerkszeugs', aufgrund der nur auf Interesse fußenden Teilnahme und aufgrund seines relativen Expertenstatus verändert sich in diesem Kontext der Status des Nutzers. Das soll noch etwas anhand der auf der schweizerischen citizen-science-Seite präsentierten Projekts Dialäkt-Äpps diskutiert werden, weil die an diesem Ort explizit als ein citizen science-Projekt geführt wird. Im Kern begründet wird das darin, dass in der Nutzung und Auswertung der App eine Art Win-win-Situation für Profi- und Laien-Wissenschaft entstehe. Das liest sich dann so: 
Unsere kostenlosen Äpps erlauben eine geographische Lokalisierung Ihres Schweizer Dialekts nach sprachwissenschaftlichen Standards. Sie können ihren Dialekt aufnehmen und die Dialekte anderer Benutzer sowie historische Aufnahmen anhand einer interaktiven Karte anhören. Gleichzeitig erlauben es die Dialäkt Äpps, zeitgenössische Schweizer Dialekte als wertvolles kulturelles Gut zu archivieren.

(https://www.schweizforscht.ch/projekte/projektarchiv/dialaekt-aepps/dialaekt-aepps; letzter Zugriff 01.06.2021)

In einem der Teilbereiche der Dialäkt-Äpps gilt die Belohnung, die eigene gelieferte Spracheingabe zugeordnet zu bekommen und durch eigenes Wissen ( $\mathrm{Zu}$ ordnung, Transkription) bereits verbessern zu können, als incentive für die Beteiligung, die als bürgerwissenschaftlich ausgewiesen wird. Über das ,Mitforschen' heißt es dort:

Wie können Bürger mitforschen? Indem die BenutzerInnen Ihren Dialekt, Geschlecht und Alter angeben, können wir neue Dialektkarten aufbereiten und diese mit historischen Daten vergleichen. Dies wiederum erlaubt uns Dialektwandel zu dokumentieren.

(https://www.schweizforscht.ch/projekte/projektarchiv/dialaekt-aepps; letzter Zugriff 01.06.2021)

Das klingt zweifellos inhaltlich nicht so furchtbar interaktiv, und jedenfalls sind das Forschungsziel und auch die Forschungsfrage vom Inneren der professionellen Wissenschaft gesetzt. Nachdenklicher in dieser Hinsicht klingt der Text für eine der Apps, die an die genannten spielerischen Tendenzen anschließt:

Bürger/-innen können zum einen mitforschen, indem sie an unserem Online-Spiel teilnehmen. Zum anderen können Ausschnitte aus den Hörbeispielen transkribiert, d.h. verschriftlicht werden. Daneben gibt es die Möglichkeit, im moderierten Forum mitzudiskutieren.

(https://www.schweizforscht.ch/projekte/projektarchiv/tour-de-suisse-din-dialaekt-tonaccent; letzter Zugriff 18.03.2020)

Wenn man nicht über Benennungen streiten will, kann man jedenfalls festhalten, dass die methodischen Möglichkeiten, die mit den neuen Medien und ihrer natürlichen Nutzung verbunden sind, eine Interaktion zwischen den Partnern der Forschungssituation ermöglicht, die viel mehr auf einer gleichen Ebene stattfinden kann, als das in traditionelleren Verfahren möglich war. Für das, was in der Sprechweise der citizen science Umgebung die Sensoren-Funktion des bürgerwissenschaftlichen Tuns genannt wird, ergeben sich hier durchaus Optionen, die einen qualitativen Schritt in der Zusammenarbeit zwischen der 
professionalisierten Wissenschaft und den Laien, aber auch Experten in bestimmten Bereichen betrifft. Viele Aussagen wird man unter diesen Bedingungen kaum mehr ohne den Einbezug großer Datenmengen, die dem Zufallsprinzip ein erhöhtes Gleichgewicht verdanken, treffen wollen. Und natürlich haben damit die Laien, die Gewährspersonen, die Experten einen unmittelbareren Einfluss im Forschungsverlauf. Allerdings wird ihr generelles Interesse an der Sache, oder vielleicht auch nur an der App, im Sinne eines vorgegebenen Forschungsdesigns und eigentlich auch im Rahmen bestimmter Forschungsparadigmata genutzt. Wie an den populäreren Beispielen angedeutet, nimmt zumindest traditionell das Laieninteresse eine eigene, in irgendeiner Weise spezifischere, in einem weiten Sinn angewandte Richtung, in der Wissen aus der jeweiligen Fachwissenschaft als Transferbasis genutzt wird. Allerdings ist diese Formulierung insofern nicht ganz adäquat, als in gewissem Umfang der Transfer als ein Rückkopplungsprozess verstanden werden kann und muss. Und das gilt nicht nur ganz banal dadurch, dass in der gemeinsamen Leistung der Datensammlung und -klassifikation zumindest die Menge der Beteiligten gegenüber den Forschenden einen empirischen Druck erzeugt, dem nicht so leicht $\mathrm{zu}$ entgehen ist wie in einer einzelnen Erhebungssituation. Es besteht eine deutliche Korrelation zwischen Forschungsparadigmen und der Wirksamkeit der Datenerzeugenden, allerdings eher als Argument der unwiderstehlichen gemeinsamen Richtung von Daten, in der crowd, ohne dass das mit dem Konzept von Schwarmintelligenz gleichzusetzen wäre. Es geht vielmehr um den Druck der besser zusammengestellten Daten auf die intelligente wissenschaftliche Analyse. In dieser Argumentation gehört die zweifellos vorhandene qualitative Veränderung der Forschungsparadigmen im Bereich der Dokumentation des Sprachgebrauchs eher zu den Folgen oder Begleitprozessen mit großen elektronischen Datenmengen.

\section{Vielleicht geht es um etwas Anderes}

Aber was man sich ja eigentlich von citizen science (proper) erwartet, sind Herangehensweisen, die mit den Ideen wissenschaftlicher Erkenntnis kompatibel sind, aber den, wenn man so will ,bürgerlichen' Gesichtspunkt zeigen. Bisher sehen wir hauptsächlich Erweiterungen, die eigentlich klassisch wissenschaftliche Beteiligung bei den sogenannten Bürgern ermöglichen, und gerade auch welche, die auf eine klassisch wissenschaftliche Fragestellung zielen. Es ist sicher nicht schlecht, wenn auf diese Weise die gemeinhin als etwas sperrig betrachtete linguistische Sichtweise in allgemeinere Diskurse eingebettet wird, 
aber eigentliche in einem an der professionalisierten Wissenschaft orientierten Sinn als wissenschaftlich angesehene Neuansätze sind zumindest in diesem Bereich nicht so recht zu sehen.

Das hat einerseits sicher damit zu tun, dass die Konzepte und Praktiken rund um citizen science doch noch neu sind, sodass sich erst Regularitäten und Modelle entwickeln müssen, die dieser Entwicklung das eigenständige Gesicht geben, das durch den Begriff nahegelegt wird. Andererseits sind die prinzipiellen analytischen Herangehensweisen gerade bei der Sprache und ihrem Gebrauch auch deswegen recht unterschiedlich, weil der Umgang mit Sprache schwer vom alltäglichen Leben abzulösen ist. Vor allem die eigene Sprache ist hauptsächlich ein funktionierender und konstitutiver Teil der gesellschaftlichen Existenz, dessen ,normales' Funktionieren kaum in den Fokus des damit nicht professionell Beschäftigten tritt. In Umkehrung der bisher besprochenen Beispiele kann man das im unterschiedlichen Status sehen, den in diesen beiden Diskurswelten normative und sprachkritische Konzepte spielen. Es gibt ein recht hohes Interesse in der Öffentlichkeit für Fragen dieses Typs, die, wenn sie denn in der professionellen Sprachwissenschaft überhaupt als wissenschaftliche Fragestallungen akzeptiert werden, in unterschiedlichen Paradigmen spielen (vgl. z. B. Eichinger 2009: 202-203). Bürgerwissenschaft ist vermutlich nicht nur stärker an einer Kritik der Praxis interessiert, sondern meistert sie oft auch (durchaus im Sinn von Sennett 2008: 45-46), und es ist sicher auch der Mühe der Wissenschaft wert, dort einen aufgeklärten Dialog zu suchen und zu bestreiten.

\section{Literaturverzeichnis}

Besch, Werner (1997): Ortssprachenforschung am Beispiel Erp. Kritische Bilanz nach (gut) 20 Jahren. In Arno Ruoff \& Peter Löffelad (Hrsg.), Syntax und Stilistik der Alltagssprache, 177180. Tübingen: Niemeyer.

Brandstetter, Alois (1982): Über den grünen Klee der Kindheit. Salzburg, Wien: Residenz.

Brinckmann, Caren, Stefan Kleiner, Ralf Knöbl \& Nina Berend (2008): German Today: an areally extensive corpus of spoken Standard German. Proceedings 6th International Conference on Language Resources and Evaluation (LREC 2008). Marrakesch, Marokko. European Language Resources Association (ELRA), 2008, 3185-3191.

Eichhoff, Jürgen (1977-2000): Wortatlas der deutschen Umgangssprachen. Vol. I/II: Bern: Francke; 1977/78, Vol. III: München: Saur 1993, Vol. IV: München: Saur 2000 III.

Eichinger, Ludwig M. (2009): Vom rechten Deutsch. Wer darf die Sprache kritisieren? In WolfAndreas Liebert \& Horst Schwinn (Hrsg.), Mit Bezug auf Sprache. Festschrift für Rainer Wimmer, 201-217. Tübingen: Narr. 
Eichinger, Ludwig M. (2010): Kann man der Selbsteinschätzung von Sprechern trauen? In Christina Ada Anders, Markus Hundt \& Alexander Lasch (Hrsg.), Perceptual Dialectology. Neue Wege der Dialektologie, 433-449. Berlin, New York: De Gruyter.

Eichinger, Ludwig M. (2016): Praktiken: etwas Gewissheit im Geflecht der alltäglichen Welt. In Arnulf Deppermann, Helmuth Feilke \& Angelika Linke (Hrsg.), Sprachliche und kommunikative Praktiken, VI-XIII. Berlin, Boston: De Gruyter.

Eichinger, Ludwig M. (2018): Korpuslinguistik im Alltag linguistischer Forschung. In Joachim Gessinger, Angelika Redder \& Ulrich Schmitz (Hrsg.), Korpuslinguistik, 69-82. Duisburg: Universitätsverlag Rhein-Ruhr.

Finke, Peter (2016): Citizen Science und die Rolle der Geisteswissenschaften für die Zukunft der Wissenschaftsdebatte. In Kristin Oswald \& René Smolarski (Hrsg.), Bürger - Künste Wissenschaft: Citizen Science in Kultur- und Geisteswissenschaften, 31-56. Gutenberg: Computus.

FOLK (Forschungs- und Lehrkorpus gesprochener Sprache). http://agd.idsmannheim.de/folk.shtml (letzter Zugriff 18.03.2020).

Hundt, Markus, Nicole Palliwoda \& Saskia Schröder (Hrsg.) (2017): Der deutsche Sprachraum aus der Sicht linguistischer Laien: Ergebnisse des Kieler DFG-Projektes. Berlin, Boston: De Gruyter.

Jakobson, Roman (2019): Linguistik und Poetik. In Ludger Hoffmann (Hrsg.), Sprachwissenschaft. Ein Reader, 170-191. Berlin, Boston: De Gruyter.

Kaiser, Julia (2018): Zur Stratifikation des FOLK-Korpus: Konzeption und Strategien. Gesprächsforschung 19, 515-552. www.gespraechsforschung-ozs.de (letzter Zugriff 01.06.2021).

Kleiner, Stefan (2015): "Deutsch heute" und der Atlas zur Aussprache des deutschen Gebrauchsstandards. In Roland Kehrein, Alfred Lameli \& Stefan Rabanus (Hrsg.), Regionale Variation des Deutschen. Projekte und Perspektiven, 489-518. Berlin, Boston: De Gruyter. Leemann, Adrian, Marie-José, Kolly, Ross Purves, David Britain \& Glaser Elvira (2016): Crowdsourcing Language Change with Smartphone Applications. PLoS ONE 11 (1). https://doi.org/10.1371/journal.pone.0143060 (letzter Zugriff 01.06.2021).

Leemann, Adrian, Curdin Derungs \& Stephan Elspaß (2019): Analyzing linguistic variation and change using gamification web apps: The case of German-speaking Europe. PloS one. 14. https://dx.plos.org/10.1371/journal.pone.0225399 (letzter Zugriff 01.06.2021).

Plewnia, Albrecht \& Astrid Rothe (2012): Sprache - Einstellungen - Regionalität. In Ludwig M. Eichinger, Albrecht Plewnia, Christiane Schoel \& Dagmar Stahlberg (Hrsg.), Sprache und Einstellungen. Spracheinstellungen aus sprachwissenschaftlicher und sozialpsychologischer Perspektive. Mit einer Sprachstandserhebung zum Deutschen von Gerhard Stickel, 9-118. Tübingen: Narr.

Purschke, Christoph (2018): Sprachliche Vielfalt entdecken mit der Lingscape-App. Der Deutschunterricht 4/2018, 70-76.

Sennett, Richard (2008): The Craftsman. London: Penguin.

Weber, Max (2002): Wissenschaft als Beruf. In ders.: Schriften 1894-1922, ausgewählt v. Dirk Kaesler, Stuttgart: Kröner 2002. https://www.molnut.unikiel.de/pdfs/neues/2017/Max_Weber.pdf (letzter Zugriff 01.06.2021).

Wittgenstein, Ludwig (1977): Vermischte Bemerkungen. Frankfurt am Main: Suhrkamp. 
